Elsevier Editorial System(tm) for Optical Switching and Networking

Manuscript Draft

Manuscript Number:

Title: OBS Contention Resolution Performance

Article Type: Research Paper

Section/Category:

Keywords: Optical burst switching, wavelength contention resolution, burst segmentation, burst deflection, limited wavelength conversion, reduced-load approximation.

Corresponding Author: Andrew Zalesky,

Corresponding Author's Institution: University of Melbourne

First Author: Andrew Zalesky

Order of Authors: Andrew Zalesky; Hai L Vu; Zvi Rosberg; Eric W. M. Wong; Moshe Zukerman

Manuscript Region of Origin:

Abstract: In this paper, a performance comparison of resolution policies specific to optical burst switching is presented. A framework, based on a reduced-load approximation, is developed to estimate path blocking probabilities in an optical burst switching network of arbitrary topology, in which any combination of the following resolution policies is in place: burst deflection, burst segmentation and limited wavelength conversion. The framework is used to compare the relative performance of each resolution policy for two sets of ingress and egress router pairs 
defined on the NSF network. With respect to the assumptions considered, it is shown that limited wavelength conversion is more effective in reducing blocking relative to burst deflection if the maximum wavelength conversion radius is sufficiently large, otherwise, burst deflection is more effective. Furthermore, limited wavelength conversion or burst deflection are more effective in reducing blocking relative to burst segmentation. Burst segmentation is justified as a stand alone resolution policy, however, using burst segmentation to complement another resolution policy offers only a marginal reduction in blocking. Both just-in-time and just-enough-time scheduling is analyzed. Simulations are implemented to corroborate the accuracy of the framework and extensions. 


\title{
OBS Contention Resolution Performance
}

\author{
Andrew Zalesky, Hai Le Vu, Zvi Rosberg, Eric W. M. Wong and Moshe Zukerman
}

\begin{abstract}
In this paper, a performance comparison of resolution policies specific to optical burst switching is presented. A framework, based on a reduced-load approximation, is developed to estimate path blocking probabilities in an optical burst switching network of arbitrary topology, in which any combination of the following resolution policies is in place: burst deflection, burst segmentation and limited wavelength conversion. The framework is used to compare the relative performance of each resolution policy for two sets of ingress and egress router pairs defined on the NSF network. With respect to the assumptions considered, it is shown that limited wavelength conversion is more effective in reducing blocking relative to burst deflection if the maximum wavelength conversion radius is sufficiently large, otherwise, burst deflection is more effective. Furthermore, limited wavelength conversion or burst deflection are more effective in reducing blocking relative to burst segmentation. Burst segmentation is justified as a stand alone resolution policy, however, using burst segmentation to complement another resolution policy offers only a marginal reduction in blocking. Both just-in-time and just-enough-time scheduling is analyzed. Simulations are implemented to corroborate the accuracy of the framework and extensions.
\end{abstract}

Index Terms-Optical burst switching, wavelength contention resolution, burst segmentation, burst deflection, limited wavelength conversion, reduced-load approximation.

\section{INTRODUCTION}

$\mathbf{U}$ NDERTAKEN in this paper is a performance comparison of wavelength contention resolution policies specific to a state of the art unacknowledged switching technology known as optical burst switching (OBS) [4], [18]. The way in which wavelength contention is resolved has a dramatic bearing on the performance of OBS. A wavelength is said to be in contention if it cannot accommodate one or more of the bursts it is scheduled, and a resolution policy refers to the way in which wavelength contention is resolved.

The trademark features underpinning OBS are as follows. Data streams are gathered at ingress routers, sorted according to destination and grouped into variable-sized switching entities known as bursts. Consider a fully formed burst that

This work was partially supported by the Australian Research Council (ARC) and a grant from the Research Grants Council of the Hong Kong Special Administrative Region, China [RGC Ref. No. 9040928].

Part of this work was conducted while Z. Rosberg and M. Zukerman were visiting the City University of Hong Kong, Hong Kong SAR, China.

A. Zalesky, H. L. Vu and M. Zukerman are with the ARC Special Research Centre for Ultra-Broadband Information Networks (CUBIN), Department of Electrical and Electronic Engineering, The University of Melbourne, Vic. 3010, Australia; email: \{a.zalesky,h.vu,m.zukerman\}@ee.mu.oz.au. CUBIN is an affiliated program of National ICT Australia.

Z. Rosberg is with the Department of Communication Systems Engineering, Ben Gurion University, Beer-Sheva, 84105, Israel; email: rosberg@bgumail.bgu.ac.il.

E. W. M. Wong is with the Optoelectronics Research Centre, Department of Electronic Engineering, City University of Hong Kong, Hong Kong SAR, China; email: ewong@ee.cityu.edu.hk. is ready-to-go. Before the burst is sent, a control packet is generated at the ingress router and sent toward the destination to set up a lightpath. The control packet, in essence, is assigned the task of heralding the arrival of the upcoming burst. Upon its arrival at each optical cross-connect along the lightpath, the burst size and arrival time are read from the control packet and the burst is scheduled in advance to an appropriate outgoing wavelength. A wavelength is said to be busy for the period it is scheduled a burst, otherwise it is free. Scheduling involves making an advance reservation for the wavelength. The burst itself is sent after a fixed delay, referred to as an offset, equal to the total processing delay encountered by the control packet. Such an offset ensures the burst cannot overtake the control packet and effectively run-out of lightpath.

OBS can be categorized in terms of when a wavelength is reserved and when a wavelength is released, referred to as scheduling. Reservation is considered immediate if the wavelength is reserved immediately upon arrival of the control packet and delayed if reservation is delayed until a time in the future when the burst is expected to arrive. Release is considered immediate if the wavelength is released immediately upon burst departure and delayed if the wavelength is released some time later by an explicit trailing control packet. Therefore, four possible categories of scheduling are possible, of which delayed reservation with immediate release, often referred to as just-enough-time (JET) [18] scheduling, and immediate reservation with delayed release, often referred to as just-in-time (JIT) [1] scheduling, are most prevalent in the literature.

The simplest resolution policy, suited to most forms of unacknowledged switching including OBS, is to block a burst in the case that wavelength contention is encountered. The data carried by a blocked burst is dumped at the optical crossconnect immediately preceding the link in which contention is encountered. The responsibility of burst retransmission is then given to higher layer protocols. Path blocking probability is defined as the stationary probability of the event in which a burst traversing a given path is blocked as a consequence of wavelength contention. Because of the unacknowledged nature of OBS, path blocking probability is an important performance measure.

To reduce high blocking probabilities, the following resolution policies have been suggested specifically for OBS, though in principle, they may also suit other forms of unacknowledged switching. Each policy is described in terms of how a burst is treated in the case that it encounters wavelength contention.

- Burst deflection [2], [6], [11], [26], [30]: an attempt is made to schedule the burst to a wavelength within an alternative outgoing link if it cannot be scheduled to the preferred outgoing link. The alternative outgoing 
link forms the first link of a deflection path, which is a path ending at the same egress router as the primary lightpath. Hence, contention is resolved by completely bypassing the preferred outgoing link in which contention is encountered.

- Burst segmentation [9], [25]: the burst is segmented at the data packet level such that a single contiguous segment of the original burst remains, which can be scheduled to an appropriate wavelength within the outgoing link. The other segments are blocked. Hence, contention is resolved by pruning away segments of the burst that give rise to contention.

- Limited wavelength conversion [20], [22], [23], [29]: an attempt is made to schedule the burst to a different outgoing wavelength, which lies within a supported range of the ingoing wavelength. The extremal case is full wavelength conversion. Optical cross-connects must be equipped with wavelength converters.

Alternative resolution policies have been suggested, such as the use of fiber delay lines to buffer a contending burst, which would otherwise be blocked (see e.g. [16] and references therein), and priority schemes [19], [27].

In this paper, a framework underpinned by the classical reduced-load approximation is developed to estimate path blocking probabilities in an OBS network of arbitrary topology, where any combination of the afore described resolution policies is in place. The framework is developed such that for combinations in which more than one resolution policy is in place, the order in which polices are applied, from highest to lowest is conversion, deflection and then segmentation. In particular, an attempt is first made to schedule an arriving burst onto the preferred outgoing link using limited wavelength conversion alone. Given no wavelength is free within the range of allowable conversion of the preferred link, and if such a wavelength is free on the alternative link, the burst is deflected to that wavelength. The burst is dumped if no such wavelength is free. If during the time that the burst is being deflected or dumped, a wavelength within the range of allowable conversion of the preferred link becomes free, the burst is segmented and the remainder of the burst is sent on the preferred link.

The utility of the framework lies in its ability to generate an estimate of path blocking probabilities in a fraction of the time demanded by simulation. The framework provides a tool for telecommunications providers and vendors to conduct a performance comparison of resolution policies specific to OBS.

As a starting point, in Section II, a reduced-load approximation is developed for the resolution policy of burst deflection. This forms the foundation of the framework. A brief survey of existing approaches for evaluating the performance of OBS is also provided. Extensions to the framework are thereafter derived for the improved resolution policies of burst segmentation and limited wavelength conversion in Sections III and IV, respectively. In Section V, it is shown how the framework is modified to incorporate combinations of the three resolution policies considered in this paper, and it is shown the way in which such combinations interact. In Section VI, the framework is used to compare the relative performance of each resolution policy for two sets of ingress and egress router pairs defined on the NSF network. Simulations are implemented to corroborate the accuracy of the framework and extensions.

\section{Reduced-LOAd APPROXIMATION With BuRST DEFLECTION}

In brief, the reduced-load approximation involves using the Erlang blocking formula, or an equivalent loss model, to estimate link blocking probabilities. As it shall be seen, assuming links evolve independently of each other gives rise to a system of fixed point equations describing the functional relation between the load offered to a link and the blocking probability of all preceding links. The fixed point, which represents a consistent set of link blocking probabilities, is found with a repeated substitution algorithm. Based on link blocking probabilities, path blocking probabilities can then be estimated by assuming blocking events occur independently from link-to-link.

The general approach underpinning the reduced-load approximation, which was popularized by Kelly [12] and Whitt [13], and subsequently advanced by Chung et al. [7], has remained a cornerstone of network performance evaluation for several decades now. For example, Barry and Humblet [3], Birman [5], Widjaja [14], [28], Kovačević and Acampora [15], and Sridharan and Sivarajan [24] have developed models based on the reduced-load approximation to evaluate the performance of an optical network. Rosberg et al. [19] recently developed a reduced-load approximation to estimate path blocking probabilities in OBS networks with full wavelength conversion. The performance of burst segmentation and various priority schemes was also analyzed. The reduced-load approximation presented in [19], which forms the foundation of the framework developed in this paper, is now extended to the resolution policy of burst deflection. Thereafter, burst segmentation and limited wavelength conversion are incorporated into the framework. Extending the framework as such facilitates the performance evaluation of any combination of the three resolution policies considered in this paper.

Burst deflection involves making an attempt to schedule a burst to a wavelength within an alternative outgoing link if it cannot be scheduled to the preferred outgoing link. By preferred outgoing link, it is meant the link associated with the shortest hop lightpath from the ingress to the egress router, which is referred to as the primary lightpath. The alternative outgoing link forms the first link of a deflection path, which is a path ending at the same egress router as the primary lightpath. The deflection scheme that is considered in this paper is such that a single deflection path is defined for each optical cross-connect along the primary lightpath, as shown in Fig. 1. For simplicity, it is assumed the primary lightpath as well as all deflection lightpaths are link disjoint. Some network topologies may preclude the establishment of deflection paths, for example, a deflection path cannot be established for an optical cross-connect with only a single outgoing link.

As deflection paths generally increase the number of links a burst may traverse, either offset must be lengthened according to the increased processing time encountered by a 


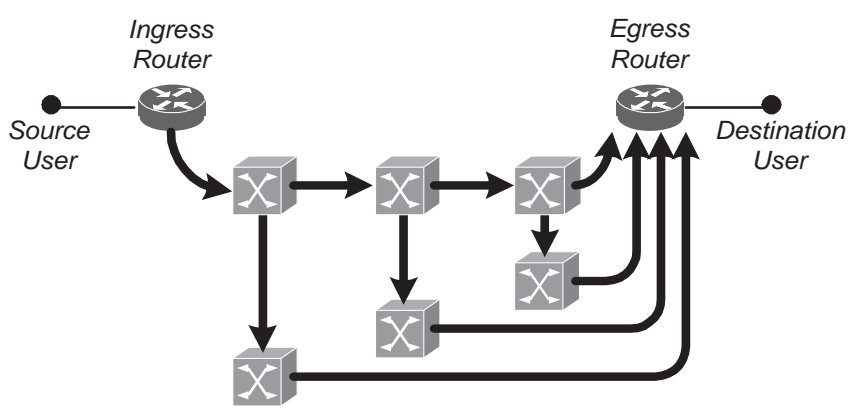

Fig. 1. Primary lightpath with deflection lightpaths

control packet traversing a deflection path. Or as suggested by Hsu et al. [11], a burst can be optically buffered at the optical cross-connect at which contention is encountered to effectively increase offset. Although the latter option mandates the deployment of high-speed optical logic and expensive fiber delay lines, it is preferred because offset is only increased if a burst is deflected. As the effect of offset is not modeled in this paper, whether the former or latter option is in place has no bearing on the framework.

Baresi et al. [2] and Wang et al. [26] have used simulation, and Chen et al. [6] and Hsu et al. [11] have developed priority queuing models for a single link to show that burst deflection may reduce blocking probabilities by several orders of magnitude, especially in lightly loaded OBS networks. Zalesky et. al [30] have analyzed alternative deflection schemes for OBS and suggested a method of stabilizing an OBS network at high loads using burst deflection.

Consider a single optical cross-connect and focus on one of its outgoing links, which comprises of $F$ fibers labeled $1,2, \ldots, F$. A fiber contains $W$ wavelengths labeled $1,2, \ldots, W$, such that wavelength $w \in\{1,2, \ldots, W\}$ is closest in distance to wavelengths $w-1$ and $w+1$, followed by wavelengths $w-2$ and $w+2$, etc., where modulo $W+1 \equiv 1$ arithmetic is assumed.

Assume that bursts arriving at the link, which may consist of newly generated external bursts arriving from an ingress router and in-progress bursts arriving from links incident to the cross-connect, form a Poisson process, where burst lengths are generally distributed. Henceforth, all time units are normalized with respect to the mean burst length. Furthermore, assume that the peak rate at which the ingress router transmits bursts is equal to the capacity of a wavelength.

To simplify the analysis considerably, the effect of offset is not modeled by effectively assigning zero offset to all bursts. This simplification is justified if the total processing time encountered by the control packet is negligible. It may be noted that a burst can be administered greater priority by increasing its offset, but doing so comes at the cost of increased delay. That is, increasing a burst's offset means that its control packet attempts to make a reservation earlier, reducing the probability of blocking of that burst.

As usual, load is measured in Erlangs, and for JET scheduling is simply equal to the mean burst arrival rate. For JIT scheduling, load must take into account the over-provisioning of wavelength resources. For details, the reader is referred to
[19].

Let $\theta$ be the external burst load offered to the link, and let $\rho_{i}, i \in\{1,2, \ldots, W\}$, be the in-progress burst load offered to wavelength $i$ within the link. An in-progress burst is restricted to a specific wavelength, that is the wavelength it arrived on, because of the wavelength continuity constraint, hence the need to specify the in-progress burst load offered to each wavelength. In practice, an external burst can be scheduled to any free outgoing wavelength within the link. However, to simplify the analysis, it is assumed that an external burst is offered to wavelength $i$, with probability $p_{i}$, independent of the distribution of free wavelengths within the link, where $\sum_{i=1}^{W} p_{i}=1$. Therefore, the external burst load offered to wavelength $i$ is $p_{i} \theta$, meaning the total load offered to wavelength $i$ is $p_{i} \theta+\rho_{i}$.

Let $L_{i}$ be the stationary probability that an external burst or an in-progress burst offered to wavelength $i$ is blocked. By modelling each wavelength as an $M / G / F / F$ queue, the set of probabilities $\left\{L_{i}: i \in 1,2, \ldots, W\right\}$ is computed by simply using the Erlang blocking formula ${ }^{1}$, which is given by

$$
L_{i}=\frac{\left(p_{i} \theta+\rho_{i}\right)^{F} / F !}{\sum_{f=0}^{F}\left(p_{i} \theta+\rho_{i}\right)^{f} / f !} .
$$

Thus, the link blocking probability of an external burst is estimated by $\sum_{i=1}^{W} p_{i} L_{i}$, while the link blocking probability of an in-progress burst arriving on wavelength $i$ is estimated by $L_{i}$. It may be noted that given the uniform distribution $p_{i}=1 / W, i \in\{1,2, \ldots, W\},(1)$ can simplified by omitting the index $i$. It may be noted that (1) assumes the load offered to each link is Poisson distributed when in fact it is smoother than a Poisson process, that is, peakedness less than one. Taking into account higher moments of the distribution of the load offered to each link may offer improved accuracy relative to (1), which only takes into account the first moment. However, doing so may add complexity to the framework.

Now consider $M$ of the afore described links labeled $1,2, \ldots, M$, which have been arbitrarily interconnected via optical cross-connects to form a network. Let $\mathbf{r}=$ $\left(r_{1}, r_{2}, \ldots, r_{|\mathbf{r}|}\right)$ be an ordered set of $|\mathbf{r}|$ links defining a path from an ingress router to an egress router, and let $\mathcal{R}$ be the set of all such paths. For each primary path $\mathbf{r}=$ $\left(r_{1}, r_{2}, \ldots, r_{|\mathbf{r}|}\right) \in \mathcal{R}$, let $\mathbf{d}\left(r_{l}\right)=\left(d_{1}, d_{2}, \ldots, d_{\left|\mathbf{d}\left(r_{l}\right)\right|}\right), l=$ $1,2, \ldots,|\mathbf{r}|$, be an ordered set of $\left|\mathbf{d}\left(r_{l}\right)\right|$ links defining a deflection path, where $d_{1}$ is an alternative outgoing link to the preferred outgoing link $r_{l}$. For simplicity, it is assumed

$$
\mathbf{r} \cap \bigcap_{l=1}^{\left|\mathbf{d}\left(r_{l}\right)\right|} \mathbf{d}\left(r_{l}\right)=\emptyset,
$$

that is, the primary path as well as all alternative paths are link disjoint.

A control packet first attempts to schedule its burst to the preferred outgoing link $r_{l}$, but if the appropriate wavelength within link $r_{l}$ is in contention, an attempt is then made to

\footnotetext{
${ }^{1}$ The Erlang blocking formula is numerically computed with the recursion $f_{n}(\rho)=\frac{\rho f_{n-1}(\rho)}{n+\rho f_{n-1}(\rho)}, n=1,2, \ldots, f_{0}=1$, where $f_{n}(\rho)$ is the stationary blocking probability given $n$ servers are offered a load $\rho$.
} 
deflect the burst by scheduling it to link $d_{1}$. The burst then traverses the deflection path $\mathbf{d}\left(r_{l}\right)$ until it is either blocked, because it cannot be scheduled to a subsequent link within the deflection path, or arrives at the egress router.

When necessary, an additional index $m \in\{1,2, \ldots, M\}$ is appended to the notation thus far defined to distinguish amongst links.

An estimate of the probability that a burst is blocked before it arrives at the egress router, referred to as the path blocking probability, is derived by assuming links evolve independently of each other. Because blocking events therefore occur independently from link-to-link, the path blocking probability of $\mathbf{r} \in \mathcal{R}$, denoted by $L_{r}$, is given by (2), which is shown below, where $\theta_{\mathbf{r}}$ is the external burst load offered to $\mathbf{r} \in \mathcal{R}$, and where the indicator

$$
I_{r, \mathbf{r}}(m)= \begin{cases}1, & m \in \mathbf{r}-\left(r, \ldots, r_{|\mathbf{r}|}\right) \\ 0, & m \in\left(r, \ldots, r_{|\mathbf{r}|}\right) .\end{cases}
$$

The indicator equals one if and only if link $r \in \mathbf{r}$ strictly precedes link $m$ in the ordered set $\mathbf{r}=\left(r_{1}, r_{2}, \ldots, r_{|\mathbf{r}|}\right)$..

Equation (2) differs from its counterpart in [19] only by addition of the term

$$
\sum_{i=1}^{W} p_{i} \sum_{r \in \mathbf{r}} L_{i}(r) \prod_{k \in \mathbf{r}}\left(1-L_{i}(k) I_{r, \mathbf{r}}(k)\right) \prod_{d \in \mathbf{d}(r)}\left(1-L_{i}(d)\right),
$$

which is the sum over all $r \in \mathbf{r}$ of the probabilities of the event in which a burst cannot be scheduled to link $r$, but is not blocked because it can be scheduled to all links along the deflection path $\mathbf{d}(r)$. In particular, given a burst is assigned to wavelength $i$, which occurs with probability $p_{i}$ : $\prod_{k \in \mathbf{r}}\left(1-L_{i}(k) I_{r, \mathbf{r}}(k)\right)$ is the probability that the burst is scheduled to all links up to, but not including link $r ; L_{i}(r)$ is the probability that the burst cannot be scheduled to link $r$ because of wavelength contention; and, $\prod_{d \in \mathbf{d}(r)}\left(1-L_{i}(d)\right)$ is the probability that after being deflected at link $r$, a burst is scheduled to all links along the deflection path $\mathbf{d}(r)$, hence arriving at the egress router.

The external burst load offered to each link is given at the outset, so are the probabilities $p_{i}$, but the in-progress burst load is functionally related to the link blocking probabilities. This functional relation is given by (3), which is shown below. To simplify the derivation of (3), it has been assumed that bursts offered to a deflection path form a Poisson process, hence the total load offered to a link is simply the superposition of the loads offered by primary and deflection paths. The error introduced by this assumption is quantified in Section VI through simulation.

The first term in (3) is the sum of the reduced-load offered by primary paths $\mathbf{r} \in \mathcal{R}$ traversing link $m$, while the second term is the sum of the reduced-load offered by deflection paths. As it has been assumed that bursts offered to a deflection path form a Poisson process, both the first and second term can be added to give the required expression.

Equation (3) reflects the fact that load is gradually thinned as it propagates along a path because of blocking. The amount by which load is thinned depends on the link blocking probabilities, and the link blocking probabilities in turn depend on the amount by which load is thinned. As such, (1) and (3) give rise to a set of fixed point equations, which is efficiently solved by repeated substitution, as specified in Algorithm 1.

Let $L_{i, n}(m)$ and $\rho_{i, n}(m), i \in\{1,2, \ldots W\}, m \in$ $\{1,2, \ldots, M\}$, be the link blocking probabilities and the inprogress burst loads, respectively, at iteration $n=0,1,2, \ldots$. of Algorithm 1.

\section{Algorithm 1: RePEATED SUbSTItUTION}

0. Initialize: Set $L_{i, 0}(m), i \in\{1,2, \ldots, W\}$, $m \in\{1,2, \ldots, M\}$, to an arbitrary distribution.

1. Compute: Set $n=n+1$. Compute $\rho_{i, n}(m), i \in\{1,2, \ldots, W\}, m \in$ $\{1,2, \ldots, M\}$, as given by (3) using $L_{i, n-1}(m)$.

2. Update: Update the link blocking probabilities $L_{i, n}(m), i \in\{1,2, \ldots, W\}$, $m \in\{1,2, \ldots, M\}$, as given by (1) using $\rho_{i, n}(m)$. Terminate if $\mid L_{i, n}(m)$ $L_{i, n-1}(m) \mid<\epsilon$, for sufficiently small $\epsilon$ for all $m \in\{1,2, \ldots, M\}$.

3. Loop: Go to step 1.

Upon termination of Algorithm 1, path blocking probabilities can be estimated as given by (2).

The reduced-load approximation that has been developed forms the foundation of the framework presented in this paper. In the next section, the resolution policy of burst segmentation is incorporated into the framework.

\section{Burst SEgmentation}

A burst consists of several hundreds of packets. With native OBS, a blocked burst must dump all of its packets, even if the proportion of contending packets is small relative to the total number of packets within a burst. Burst segmentation is underpinned by the concept of segmenting a burst at the packet level such that a single contiguous segment of the original burst remains. The remaining segment, which would be otherwise be blocked, is scheduled as usual. Thus, burst segmentation effects a reduction in packet blocking probability.

As the effect of offset is not modeled in this paper, it suffices to divide a burst that encounters contention into two contiguous segments, each of which contain an integer number of packets. It may be noted that if the effect of offset is taken into account, dividing a burst into more than two segments may be required. Specifically, the remaining burst segment may require further segmentation for it may contain packets that contend with an existing reservation made in advance for a burst with sufficiently long offset. In other words, a burst with sufficiently long offset may preempt the remaining burst segment.

Vokkarane et al. [25] used simulation to quantify the performance of burst segmentation in a hypothetical OBS network. Detti et al. [9] developed an analytical model for a single link to quantify the reduction in packet blocking probability achievable with burst segmentation. As in [19], the model presented in [17] is used to estimate link blocking probabilities in the same way as the Erlang blocking formula was used in 


$$
\begin{aligned}
L_{r}= & 1-\sum_{i=1}^{W} p_{i}\left(\prod_{r \in \mathbf{r}}\left(1-L_{i}(r)\right)+\sum_{r \in \mathbf{r}} L_{i}(r) \prod_{k \in \mathbf{r}}\left(1-L_{i}(k) I_{r, \mathbf{r}}(k)\right) \prod_{d \in \mathbf{d}(r)}\left(1-L_{i}(d)\right)\right) \\
\rho_{i}(m)= & \sum_{\mathbf{r} \in \mathcal{R}: m \in \mathbf{r}} \theta_{\mathbf{r}} p_{i} \prod_{r \in \mathbf{r}}\left(1-L_{i}(r) I_{r, \mathbf{r}}(m)\right) \\
+ & \sum_{\mathbf{r} \in \mathcal{R}} \sum_{r \in \mathbf{r}: m \in \mathbf{d}(r)} \theta_{\mathbf{r}} p_{i} L_{i}(r) \prod_{k \in \mathbf{r}}\left(1-L_{i}(k) I_{r, \mathbf{r}}(k)\right) \prod_{d \in \mathbf{d}(r)}\left(1-L_{i}(d) I_{d, \mathbf{d}(r)}(m)\right) \\
& i \in\{1,2, \ldots, W\}, m \in\{1,2, \ldots, M\}
\end{aligned}
$$

Section II. According to the model presented in [17], (1) is replaced with $L_{i}=E\left(H_{i}\right) /\left(p_{i} \theta+\rho_{i}\right), i \in\{1,2, \ldots, W\}$, where $E\left(H_{i}\right)$ is the mean loss rate, which is given by

$$
E\left(H_{i}\right)=\sum_{n=1}^{\infty} \frac{n\left(p_{i} \theta+\rho_{i}\right)^{n+F} e^{-\left(p_{i} \theta+\rho_{i}\right)}}{(n+F) !} .
$$

Equation (4) is derived by extending the $M / G / F / F$ queue used to model each wavelength in Section II to an $M / G / \infty$ queue with an unlimited number of pseudo-wavelengths in addition to the original $F$ wavelengths. Pseudo-wavelengths have no physical interpretation, however, as it shall be seen, they are a convenient modeling device to represent bursts that are dumping packets.

The $M / G / \infty$ queue can be thought of in terms of the behavior of the original $M / G / F / F$ queue if the number of busy wavelengths is less than or equal to $F$. Otherwise, if $F+i, i=1,2, \ldots$, wavelengths are busy, $i$ of the $F+i$ busy wavelengths can be thought of as pseudo-wavelengths that must dump packets. The remaining $F$ wavelengths represent real wavelengths. Thus, if $i$ pseudo-wavelengths are busy, $i$ out of every $F+i$ packets are dumped. In other words, for every $F$ packets sent on the $F$ real wavelengths, $i$ packets are dumped by the $i$ pseudo-wavelengths. From the instant one of the $F$ real wavelengths becomes free, it begins to serve the remaining segment of the burst scheduled to the first pseudowavelength.

In the next section, the resolution policy of limited wavelength conversion is incorporated into the framework. This involves developing a new model to estimate link blocking probabilities, and modifying (2) and (3) accordingly.

\section{Limited WAVELENGTH CONVERsion}

Thus far a lightpath has been constrained to the same wavelength within each link, referred to as the wavelength continuity constraint. A wavelength converter is a device that provides the ability to switch data from an incoming wavelength to a different outgoing wavelength. Wavelength conversion allows the wavelength continuity constraint to be relaxed. Therefore, honoring a lightpath request only requires some wavelength to be free within each link, whereas no wavelength conversion requires the same wavelength to be free within each link.

Wavelength converters are costly devices and bear some side effects. One such side effect is that output power strongly deteriorates as a function of the distance between the input and output wavelengths, which motivates the study of limited wavelength conversion [10]. Wavelength converters with a limited conversion range allow an incoming wavelength to be switched only to a small subset of outgoing wavelengths, referred to as the range of conversion. For example, it has been shown [8] that all-optical wavelength conversion capable of switching an incoming wavelength to two different outgoing wavelengths for a total conversion range of $20 \mathrm{~nm}$ is achievable through four wave mixing in a semiconductor optical amplifier.

Models to evaluate path blocking probabilities in an optical network using acknowledged switching and limited wavelength conversion have been presented by Tripathi and Sivarajan [23], Sharma and Varvarigos [22] and Yates et al. [29]. They concluded that limited wavelength conversion with a small range is usually sufficient to equal the performance of full wavelength conversion. Recently Rosberg et al. [21] presented a model to estimate path blocking probabilities in an OBS network with either JET or JIT scheduling for the two wavelength conversion policies described below. In this paper, the model presented in [21] is incorporated into the framework with burst deflection and segmentation.

Optical cross-connects are equipped with limited wavelength converters, imposing the constraint that an in-progress burst arriving on ingoing wavelength $i$ can only be converted to an outgoing wavelength

$$
j \in N^{i}=\{j:|j-i| \leq d\}, \quad \forall i \in\{1,2, \ldots, W\},
$$

where $d$ is a small non-negative integer specifying the conversion range, referred to as the conversion radius, and modulo $W+1 \equiv 1$ arithmetic is assumed. The set $N^{i}$ is called the target range of wavelength $i$. An external burst is not subject to the constraint imposed by limited wavelength conversion.

An in-progress burst arriving on ingoing wavelength $i$ can be scheduled to any of a number of free outgoing wavelengths within target range $N^{i}$. A wavelength conversion policy specifies how to select one such free outgoing wavelength. Two wavelength conversion policies were considered in [21], they are as follows.

- Random (R), where wavelengths within a target range are first randomly ordered and the first free wavelength is selected.

- Nearest Wavelength First (NWF), where a wavelength within the target range closest in distance to the incoming 
wavelength is selected. A coin is flipped if there are two free wavelengths equal in distance to the incoming wavelength.

A burst is blocked if all wavelengths within its target range are busy. Policy NWF gives preference to outgoing wavelengths closer in distance to the incoming wavelength, reflecting the fact that output power strongly deteriorates as a function of the distance between the ingoing and outgoing wavelengths.

It has been assumed in [21] that an external burst is allocated to target range $N^{i}, i \in\{1,2, \ldots, W\}$, with probability $p_{i}$, independent of the distribution of free wavelengths within the link. Policy R is then used to select a free outgoing wavelength within the randomly selected target range. Therefore, the external burst load offered to target range $N^{i}$ is $p_{i} \theta$, meaning the total load offered to target range $N^{i}$ is $\rho_{i}+p_{i} \theta$.

To incorporate limited wavelength conversion into the framework, the model presented in [21] is used to estimate link blocking probabilities, which replaces the Erlang blocking formula in the reduced-load approximation. The building block used in this paper is the stationary probabilities of the following Markov process. For every wavelength $i$, let $X_{i}(t)$ be the number of bursts scheduled to wavelength $i$ at time $t$, and let $\mathbf{X}^{i}(t)=\left\{X_{k}(t): k \in N^{i}\right\}, \quad i \in\{1,2, \ldots, W\}$. For wavelength conversion policy $P \in\{\mathrm{R}, \mathrm{NWF}\}$, let $\Pi_{i}^{P}(\mathbf{x})$ be the stationary probability of being in state $\mathbf{X}^{i}(t)=\mathbf{x}$. An algorithm for computing $\Pi_{i}^{P}(\mathbf{x})$ is given in [21].

The link blocking probability of an external burst is estimated by

$$
\sum_{i=1}^{W} p_{i} \Pi_{i}^{P}(F, F, \ldots, F)
$$

and the link blocking probability of an in-progress burst arriving on ingoing wavelength $i$ is estimated by $\Pi_{i}^{P}(F, F, \ldots, F)$.

Once $\Pi_{i}^{P}(\mathbf{x}), i \in\{1,2, \ldots, W\}$, has been computed, path blocking probabilities can be estimated in much the same way as in Section II, however, several modifications are required to take into account the two scheduling policies.

Let $G_{i, j}^{P}(m), i, j \in\{1,2, \ldots, W\}, m \in\{1,2, \ldots, M\}$, be the probability that an in-progress burst arriving on wavelength $i$ within an arbitrary link incident to link $m$ is scheduled to wavelength $j$ within link $m$, given policy $P \in\{\mathrm{R}, \mathrm{NWF}\}$. Expressions giving $G_{i, j}^{P}(m), i, j \in\{1,2, \ldots, W\}, m \in$ $\{1,2, \ldots, M\}$, in terms of $\Pi_{i}^{P}(\mathbf{x})$ are derived in [21].

Given policy $P \in\{\mathrm{R}, \mathrm{NWF}\}$, the path blocking probability of $\mathbf{r} \in \mathcal{R}$ is

$$
1-\sum \mathbf{p G}^{\mathrm{R}}\left(r_{1}\right) \mathbf{G}^{\mathrm{P}}\left(r_{2}\right) \mathbf{G}^{\mathrm{P}}\left(r_{3}\right) \ldots \mathbf{G}^{\mathrm{P}}\left(r_{H}\right),
$$

where $\mathbf{p}=\left(p_{1}, p_{2}, \ldots, p_{W}\right)$, and

$$
\mathbf{G}^{\mathrm{P}}(m)=\left(\begin{array}{cccc}
G_{1,1}^{\mathrm{P}}(m) & G_{1,2}^{\mathrm{P}}(m) & \cdots & G_{1, W}^{\mathrm{P}}(m) \\
G_{2,1}^{\mathrm{P}}(m) & G_{2,2}^{\mathrm{P}}(m) & \cdots & G_{2, W}^{\mathrm{P}}(m) \\
\vdots & \vdots & \ddots & \vdots \\
G_{W, 1}^{\mathrm{P}}(m) & G_{W, 2}^{\mathrm{P}}(m) & \cdots & G_{W, W}^{\mathrm{P}}(m)
\end{array}\right) .
$$

The summation in equation (5) is to be understood as the summation of each of the $W$ elements of the row vector given by $\mathbf{p G}^{\mathrm{R}}\left(r_{1}\right) \mathbf{G}^{\mathrm{P}}\left(r_{2}\right) \mathbf{G}^{\mathrm{P}}\left(r_{3}\right) \ldots \mathbf{G}^{\mathrm{P}}\left(r_{H}\right)$.
The average path blocking probability is given by

$$
1-\bar{\theta} \sum_{\mathbf{r} \in \mathcal{R}} \theta_{\mathbf{r}} \sum \mathbf{p G}^{\mathrm{R}}\left(r_{1}\right) \mathbf{G}^{\mathrm{P}}\left(r_{2}\right) \mathbf{G}^{\mathrm{P}}\left(r_{3}\right) \ldots \mathbf{G}^{\mathrm{P}}\left(r_{H}\right),
$$

where $\bar{\theta}=1 / \sum_{\mathbf{r} \in \mathcal{R}} \theta_{\mathbf{r}}$.

The external burst load is given at the outset, so are the probabilities $\mathbf{p}$, but the in-progress burst load is functionally related to the stationary distributions $\Pi_{i, m}^{\mathrm{P}}, i \in\{1,2, \ldots, W\}$, $m \in\{1,2, \ldots, M\}$, and must be computed. Let $\rho_{i}(m)$, be the in-progress burst load offered to wavelength $i$ within link $m$, and let $\rho(m)=\left(\rho_{1}(m), \rho_{2}(m), \ldots, \rho_{W}(m)\right)$. Given policy $P \in\{\mathrm{R}, \mathrm{NWF}\}$,

$$
\rho(m)=\sum_{\mathbf{r} \in \mathcal{R}: r_{m} \in \mathbf{r}} \theta_{\mathbf{r}} \mathbf{p} \mathbf{G}^{\mathrm{R}}\left(r_{1}\right) \mathbf{G}^{\mathrm{P}}\left(r_{2}\right) \mathbf{G}^{\mathrm{P}}\left(r_{3}\right) \ldots \mathbf{G}^{\mathrm{P}}\left(r_{m-1}\right),
$$

$m \in\{1,2, \ldots, M\}$. Equation (6) is analogous to (3) and derived by summing the reduced load offered by all paths $\mathbf{r} \in \mathcal{R}$ traversing link $m$.

The amount by which load is thinned depends on the stationary probabilities $\Pi_{i, m}^{\mathrm{P}}, i \in\{1,2, \ldots, W\}, m \in\{1,2, \ldots, M\}$, and the stationary probabilities in turn depend on the amount by which load is thinned. As such, equation (6) gives rise to another set of fixed point equations, which can be solved using the usual repeated substitution algorithm.

\section{Combinations of Resolution Policies}

A framework was developed in the previous three sections to estimate path blocking probabilities in an OBS network in which one of the three resolution policies considered in this paper is in place. In this section, it is shown how the framework is modified to incorporate combinations of resolution policies, e.g. limited wavelength conversion in combination with burst segmentation, or burst deflection in combination with burst segmentation, and it shown how such combinations interact within the framework. Deflection and segmentation may be combined in several ways as segmentation may be restricted to either preferred links or alternative links, or no restriction may be imposed.

The framework does not cover all possible combinations; it does however cover combinations that maximize traffic on the primary lightpaths. In particular, combinations in which segmentation is combined with deflection, or a combination of deflection and conversion, if a burst is being deflected to an alternative link, as soon as a wavelength within the allowable conversion range becomes free on the preferred link the burst is segmented and the remaining segment is scheduled to the preferred link. An alternative is to continue deflecting the burst to an alternative link even though a wavelength becomes free on the preferred link, hence avoiding the shortcomings of segmentation. However, in practice primary and deflection lightpaths are determined a priori based on an optimality criterion. Therefore, it is sensible to schedule both bursts and burst segments to primary lightpaths.

The framework may address questions of the following nature. Suppose an OBS network supports limited wavelength conversion and deflection, what further reduction in path blocking probabilities can be achieved with segmentation? 
Alternatively, if limited wavelength conversion is in place, what further reduction can be achieved with deflection or segmentation, or a combination of deflection and segmentation?

The framework is modified as follows to suit a specific combination resolution policies. If burst segmentation is in place, (4) is used to estimate link blocking probabilities in the same way as the Erlang blocking formula was used in Section II. And if limited wavelength conversion is in place, link blocking probabilities are estimated based on the stationary distribution of the Markov Process $\mathbf{X}^{i}(t), i \in\{1,2, \ldots, W\}$, defined in Section IV.

For combinations in which both limited wavelength conversion and burst segmentation are in place, an infinite number of pseudo-wavelengths are 'appended' to the state of the Markov process $\mathbf{X}^{i}(t)$ in which all wavelengths within the target range are busy, that is $\mathbf{x}=(F, F, \ldots, F)$. This can be thought of as appending the $M / G / \infty$ queue used in Section III. In particular, if all wavelengths within a target range are busy an arriving burst is scheduled to the first pseudo-wavelength. That is, the process makes a transition to the new state in which all real wavelengths within the target range are busy and one pseudo-wavelength is busy. Then if another burst arrives and no bursts complete transmission the process makes a transition to the new state in which all real wavelengths within the target range are busy and two pseudo-wavelengths are busy, as shown in Fig. 2. Link blocking probabilities are estimated based on the stationary distribution of the appended $M / G / \infty$ queue as specified in Section III.

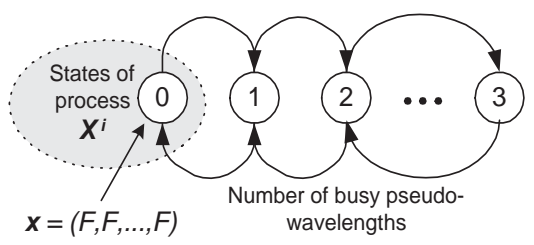

Fig. 2. $M / G / \infty$ queue defined in Section III 'appended' to the state of the Markov process $\mathbf{X}^{i}(t)$ in which all wavelengths within the target range are busy

Consider an in-progress or external burst arriving at an arbitrary link within the network. Assume the link is provided with an alternative link to facilitate burst deflection. Also assume a combination of all three resolution policies is in place. As discussed, an attempt is first made to schedule the burst onto the preferred link using limited wavelength conversion alone. Given no wavelength is free within the target range of the preferred link, and if such a wavelength is free on the alternative link, the burst is deflected to that wavelength. The burst is dumped if no such wavelength is free. If during the time that the burst is being deflected or dumped, a wavelength becomes free within the target range of the preferred link, the burst is segmented and the remainder of the burst is sent on the preferred link. It may be noted that this is consistent with OBS variants such as JIT in which the end of the existing burst is not known in advance.

The flow chart shown in Fig. 3 describes the way in which a combination of resolution policies interact as such. In Fig. 3, the decision node labeled 'Wavelength free?' is in reference to a free wavelength within the appropriate target range, which is a single wavelength if wavelength conversion is not in place. And wavelength $j$ can be a wavelength within either the preferred or alternative link that is within the appropriate target range and that becomes free before the entire burst is dumped.

The way in which a combination of resolution polices interact is not unique. The framework requires modification to cope with cases in which polices interact differently.

\section{Numerical Performance Evaluation}

The framework is used to estimate the average path blocking probability for two sets of ingress and egress router pairs defined on the NSF network. The purpose is to evaluate the relative performance of all combinations of resolution policies considered in this paper.

Discrete event simulations are implemented to quantify the error introduced by the following modeling assumptions made in developing the framework:

- Links evolve independently of each other.

- Target ranges evolve independently of each other.

- The superposition of primary and deflected bursts offered to each link forms a Poisson process.

Simulations do not quantify the error introduced by the following assumptions associated with purely physical effects:

- Wavelengths within a fiber wrap-around to form a ring.

- All bursts are assigned zero offset.

- Segmentation occurs at packet boundaries.

- Reconfiguration time of an output port of an optical crossconnect is zero.

To avoid excessive running times, simulations are implemented to generate data points giving average path blocking probabilities greater than approximately $10^{-5}$. Also, simulations of burst segmentation are not implemented due to the exorbitant amount of time required to maintain bookkeeping for each burst segment. All data points generated by simulation are shown with $95 \%$ confidence intervals. Confidence intervals are estimated by the method of batch means, where 10 batches were simulated for each data point. A stopping criterion of $10^{-10}$ was used for both repeated substitution algorithms.

The NSF network topology, which is shown in Fig. 4, consists of 32 links and 13 optical cross-connects, which may also function as ingress and egress routers. Each link consists of one fiber containing 80 wavelengths. Two sets consisting of 12 distinct ingress and egress router pairs, which are defined in Table I, are randomly selected to reflect different configurations. All ingress and egress router pairs within a set are offered the same external burst load. Given that all links are the same length, shortest paths are computed for each ingress and egress router pair with Dijkstra's algorithm.

An external burst is allocated to target range $N^{i}, i \in$ $\{1,2, \ldots, 80\}$, with probability $1 / 80$. Because of this uniform allocation of bursts, wavelength conversion polices NWF and $\mathrm{R}$ yield equal blocking probabilities and the results presented apply to both conversion policies. The reader is referred to [21] for the exact conditions ensuring both conversion policies yield equal blocking probabilities. 


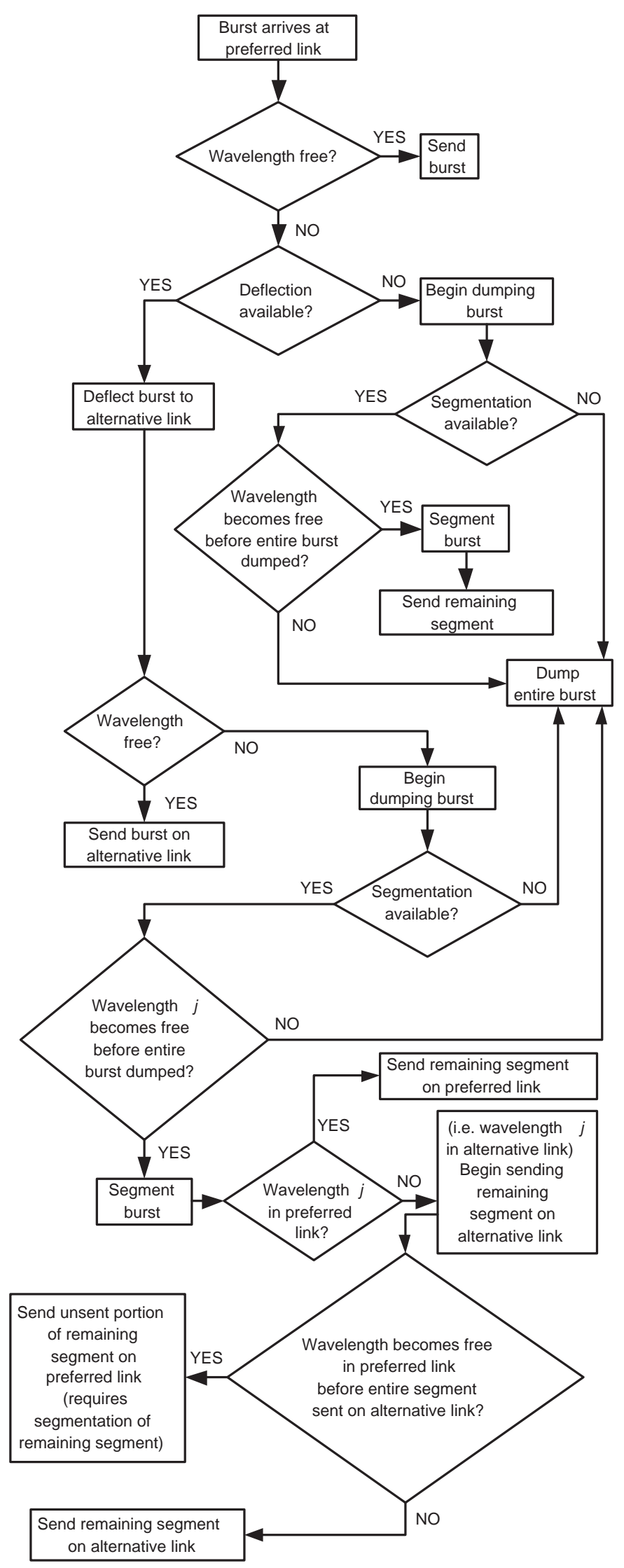

Fig. 3. Flow chart describing the way in which a combination of resolution policies interact in a single link that is provided with an alternative link

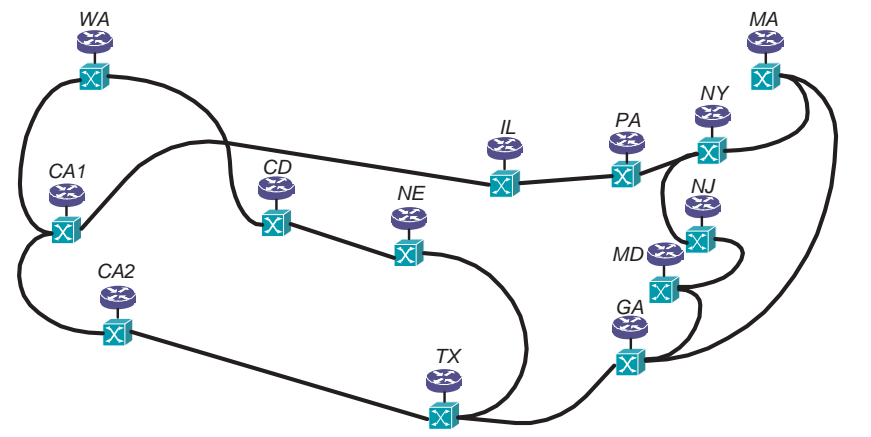

Fig. 4. NSF network topology, each solid line represents two links aligned in opposing directions

TABLE I

TWO SETS OF INGRESS AND EGRESS ROUTER PAIRS

\begin{tabular}{||c|c||c|c||}
\hline \hline \multicolumn{2}{|c||}{ Set 1 } & \multicolumn{2}{c||}{ Set 2 } \\
\hline Ingress & Egress & Ingress & Egress \\
\hline WA & MD & NJ & NE \\
CA1 & IL & IL & NY \\
CA1 & MA & CA2 & WA \\
CA2 & MA & WA & PA \\
TX & NY & CA2 & TX \\
GA & MA & CA2 & PA \\
MD & WA & CA1 & GA \\
IL & CA1 & MD & NJ \\
MA & CA1 & MA & PA \\
MA & CA2 & CA2 & CA1 \\
NY & TX & NE & GA \\
MA & GA & PA & CD \\
\hline \hline
\end{tabular}

Average path blocking probabilities are plotted against the normalized load offered to each ingress and egress router pair. To correctly compare commensurate data points generated by the framework and simulation, a measure that is not a function of blocking, such as the normalized load offered to each ingress and egress router pair, is essential. It may be noted that the normalized load offered to each ingress and egress router pair may give a very crude indication of mean link utilization. Three plots corresponding to the conversion radii $d=1,2,3$ are presented per axis. Table II shows a summary of plots.

TABLE II

SUMMARY OF PLOTS

\begin{tabular}{||c|c|c|c||}
\hline \hline Set 1 & Set2 & Segmentation & Deflection \\
\hline Fig. 6 & Fig. 10 & $\times$ & $\times$ \\
Fig. 8 & Fig. 12 & $\times$ & $\checkmark$ \\
Fig. 7 & Fig. 11 & $\checkmark$ & $\times$ \\
Fig. 9 & Fig. 13 & $\checkmark$ & $\checkmark$ \\
\hline
\end{tabular}

Observation of Figs. 6 - 13 shows that although errors introduced by modeling assumptions are not negligible, the framework is capable of generating a ballpark estimate of path blocking probabilities. In fact, it is at times difficult to discern plots generated by the framework and simulation. Error is noticeable as the conversion radius is increased, which is attributable to greater interleaving amongst target ranges, making less valid the assumption that target ranges 
evolve independently of each other. Figs. 7 and 11 confirm that assuming primary and deflected bursts form a Poisson process also introduces error. However, for all simulated data points, the error is always significantly less than one order of magnitude.

So which resolution policy is most effective? Foremost, observe that the performance of all resolution policies deteriorates with an increase in utilization. Thus, resolution policies in general offer the greatest benefit at low utilizations. As expected, the combination consisting of all three resolution policies, that is conversion and segmentation and deflection, offers superior performance in both sets of ingress and egress router pairs. However, if combinations are forbidden, that is the performance comparison is restricted to one of the three resolution policies, the following statements can be formulated.

- Limited wavelength conversion or burst deflection are more effective in reducing blocking relative to burst segmentation.

- Limited wavelength conversion is more effective in reducing blocking relative to burst deflection if the maximum wavelength conversion radius is sufficiently large, otherwise, burst deflection is more effective.

The latter statement can be interpreted as follows. Based on a comparison of the low utilization regions of Figs. 10 and 11 , burst deflection is preferable relative to limited wavelength conversion, given that the conversion radius only allows $d$ to be increased from 1 to 2 . However, limited wavelength conversion is slightly preferable relative to burst deflection, given that the conversion radius allows $d$ to be increased from 1 to 3 .

\section{A. Network Dimensioning}

A telecommunications provider or vendor may have interest in dimensioning an OBS network to minimize capacity subject to quality of service constraints. To show the utility of the framework in performing such dimensioning calculations, the minimum number of wavelengths required to ensure a blocking probability that is less than $10^{-3}$ is computed for a single link containing a single fiber. A single link provides an ideal setting to compare the relative performance of each resolution policy, as effects relating to paths of varying hops, varying link utilization, varying link sharing degrees, etc., which are present in a network setting and may mask underlying trends, are avoided.

Fig. 5 shows the minimum number of wavelengths required to ensure a blocking probability that is less than $10^{-3}$ for different combinations of resolution policies. For burst deflection, an additional single link is included to act as a deflection path.

Fig. 5 can be used in dimensioning a network as follows. For example, given a conversion radius $d=1$, and no other resolution policies, well in excess of 280 wavelength are needed to ensure a blocking probability that is less than $10^{-3}$. However, if burst segmentation is introduced about 275 wavelength are required; if burst deflection is introduced about 55 wavelength are required; and if segmentation and deflection is introduced only about 40 wavelengths are required. Also, observation of

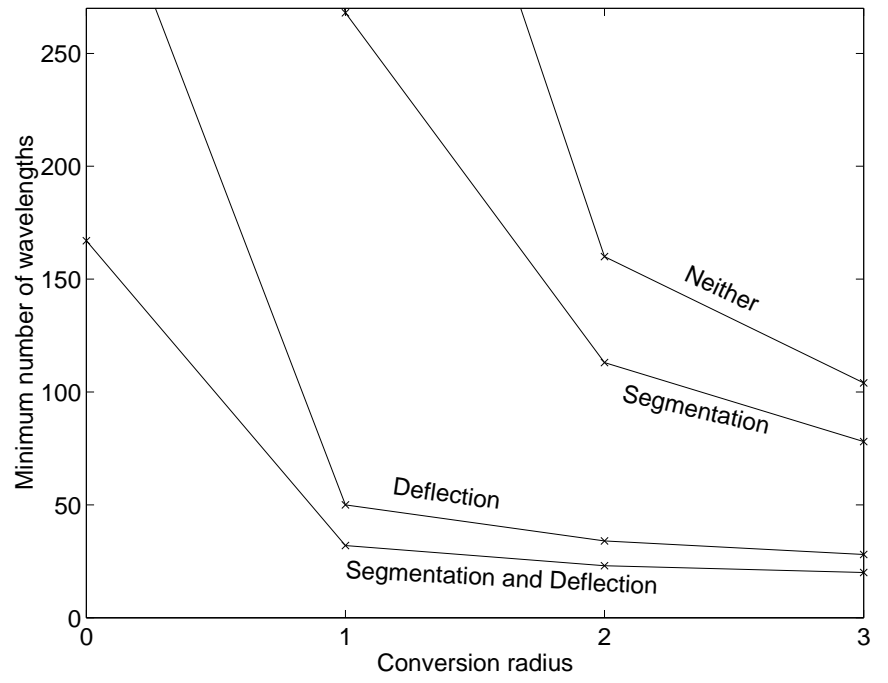

Fig. 5. Minimum number of wavelengths required to achieve a blocking probability of $10^{-3}$

Fig. 5 shows that the benefit of limited wavelength conversion is decreased as the conversion radius is increased.

Based on Fig. 5, burst segmentation is justified as a stand alone resolution policy. However, using burst segmentation to complement burst deflection, limited wavelength conversion or a combination of burst deflection and limited wavelength conversion may be unjustified as only a marginal reduction in blocking is achieved.

\section{CONCLUSION}

The framework developed in this paper was shown to provide ballpark estimates of path blocking probabilities in an OBS network of arbitrary topology, where any combination of three resolution policies is in place. The utility of the framework lies in its ability to generate an estimate in a fraction of the time demanded by simulation. From the viewpoint of telecommunications providers and vendors, the framework provides an efficient means to perform network dimensioning.

As expected, a combination of all three resolution policies considered in this paper offers superior performance. However, if combinations are forbidden, it was shown that limited wavelength conversion is more effective in reducing blocking relative to burst deflection if the maximum wavelength conversion radius is sufficiently large, otherwise, burst deflection is more effective. Advancement of wavelength conversion technology that allows for an extended conversion radius $(d \geq 4)$ may position limited wavelength conversion as the resolution policy of choice for OBS. Another advantage of limited wavelength conversion is that unlike burst deflection, it is does not require a lengthening of offset to accommodate for the increased processing delay encountered in traversing deflection paths.

It may be noted that many aspects, which are not reflected in this paper, may be of overriding importance when determining the suitability of a resolution policy for OBS. Such aspects may include complexity of control, reliability, compatibility with existing network elements and cost. 


\section{REFERENCES}

[1] I. Baldine, G. N. Rouskas, H. G. Perros, D. Stevenson, "JumpStart: A Just-in-Time Signaling Architecture for WDM Burst Switched Networks," IEEE Commun. Mag., vol. 40, Feb. 2002, pp. 82-89.

[2] M. Baresi, S. Bregni, A. Pattavina, G. Vegetti, "Deflection Routing Effectivness in Full-Optical IP Packet Switching Networks", in Proc. IEEE ICC, Anchorage, AK, May 2003.

[3] R. A. Barry, P. A. Humblet, "Models of Blocking Probability in AllOptical Networks with and without Wavelength Chnagers," IEEE J. Select. Areas in Commun., vol. 14, no. 5, June 1996, pp. 858-867.

[4] T. Battestilli and H. Perros, "An Introduction to Optical Burst Switching," IEEE Commun. Mag., vol. 41, Aug. 2003, pp. S10-S15.

[5] A. Birman, "Computing Approximate Blocking Probabilities for a Class of All-Optical Networks," IEEE J. Select. Areas in Commun. / J. Lightwave Technol., Special Issue Optical Networks, vol. 14, June 1996, pp. 852-857.

[6] Y. Chen, H. Wu, D. Hu, C. Qiao, "Performance Analysis of Optical Burst Switched Node with Deflection Routing," in Proc. IEEE ICC, Anchorage, AK, May 2003.

[7] S. Chung, A. Kashper, K. W. Ross, "Computing Approximate Blocking Probabilities for Large Loss Networks With State-Dependent Routing," IEEE/ACM Trans. Networking, vol. 1, Feb. 1993, pp. 105-115.

[8] E. Ciaramella, G. Contestabile, F. Curti and A. D'Ottavi, "Fast Tunable Wavelength Conversion for All-Optical Packet Switching," IEEE Photon. Tech. Letters, vol. 12, no. 10, Oct. 2000, pp. 1361-1363.

[9] A. Detti, V. Eramo, M. Listanti, "Performance evaluation of a New Technique for IP Support in a WDM optical Network: Optical Composite Burst Switching (OCBS)," IEEE J. Lightwave Tech., vol 20., Feb. 2002, pp. $154-165$.

[10] T. Durhuus, B. Mikkelsen, C. Joergensen, S. L. Danielsen, K. E. Stubkjaer, "All-Optical Wavelength Conversion by Semiconductor Optical Amplifiers," IEEE J. Lightwave Tech., vol. 14, no. 6, June 1996, pp. 942-954.

[11] C. F. Hsu, T. L. Liu, N. F. Huang, "Performance Analysis of Deflection Routing in Optical Burst Switched Networks," in Proc. IEEE INFOCOM, New York, NY, June 2002, pp. 55-73.

[12] F. P. Kelly, "Blocking Probabilities in Large Circuit-Switched Networks," Adv. in App. Prob., vol. 18, 1986, pp. 473-505.

[13] W. Whitt, "Blocking when Service is Required from Several Facilities Simultaneosuly," AT\&T Technical Journal, vol. 64, no. 8, Oct. 1985, pp. 339-352.

[14] A. K. Kompella, I. Widjaja, "Burst-Level Admission Control Protocols with Multirate Traffic and Arbitrary Network Topology," in Proc. 4th Inter. Conf. on Computer Commun. and Networks, Sept. 20-23, 1995, pp. $396-403$.

[15] M. Kovačević and A. Acampora, "On Wavelength Translation in AllOptical Networks," in Proc. IEEE INFOCOM, Boston, MA, April 1995, pp. 413-422.

[16] J. Li, C. Qiao, J. Xu, D. Xu, "Maximizing Throughput for Optical Burst Switching Networks," in Proc. IEEE INFOCOM, Hong Kong, China, March 2004.

[17] M. Neuts, Z. Rosberg, H. L. Vu, J. White, M. Zukrman, "Performance Analysis of Optical Composite Burst Switching," IEEE Commun. Letters, vol. 6, no. 8, Aug. 2002, pp. 346-348.

[18] C. Qiao, M. Yoo, "Optical Burst Switching (OBS): A New Paradigm for an Optical Internet," J. High Speed Nets., vol. 8, Jan. 1999, pp. 69-84.

[19] Z. Rosberg, H. L. Vu, M. Zukerman and J. White, "Performance Analyses of Optical Burst Switched Networks," IEEE J. on Select. Areas in Commun., vol. 21, Sept. 2003, pp. 1187-1197.

[20] Z. Rosberg, H. L. Vu and M. Zukerman, "Performance Evaluation of Optical Burst Switching Networks with Limited Wavelength Conversion," Proc. ONDM 2003, 7th IFIP Working Conf. on Optical Network Design and Modeling, vol. 2, Budapest, Hungary, February 2003, pp. 1155-1169.

[21] Z. Rosberg, A. Zalesky, H. L. Vu, M. Zukerman, "Limited Wavelength Conversion in OBS Networks," submitted for publication, preprint available at http://www.cse.bgu.ac.il/rosberg/papers/rzvz_05_04_lim_wave_obs.pdf

[22] V. Sharma, E. A. Varvarigos, "An Analysis of Limited Wavelength Translation in Regular All-Optical WDM Networks," IEEE J. Lightwave Tech., vol. 18, Dec. 2000, pp. 1606-1619.

[23] T. Tripathi, K. N. Sivarajan, "Computing Approximate Blocking Probabilities in Wavelength Routed All-Optical Networks with Limited-Range Wavelength Converion,” IEEE J. Select. Areas Commun., vol. 18, Oct. 2000, pp. 2123-2129.
[24] A. Sridharan, K. N. Sivarajan, "Blocking in All-Optical Networks," IEEE/ACM Trans. Networking, vol. 12, no. 2, April 2004, pp. 384-397.

[25] V. M. Vokkarane, J. P. Jue, S. Sitaraman, "Burst Segmentation: An Approach for Reducing Packet Loss in Optical Burst Switched Networks," in Proc. IEEE ICC, New York, NY, April 2002.

[26] X. Wang, H. Morikawa, T. Aoyama, "Deflection Routing Protocol for Burst Switching WDM Mesh Networks," in Proc. SPIE/IEEE Terabit Optical Networking: Architecture, Control, and Management Issues, Boston, MA, Nov. 2000, pp. 242-252.

[27] J. White, R. Tucker, K. Long, "Merit-based Scheduling Algorithm for Optical Burst Switching," Conf. on Optical Internet, COIN2002, Cheju Island, Korea, July 2002, pp.75-77.

[28] I. Widjaja, "Performance Analysis of Burst-Admission Protocols," IEE Proc. Commun., vol. 142, no. 1, Feb. 1995, pp. 7-14.

[29] J. Yates, J. Lacey, D. Everitt and M. Summerfield, "Limited Range Wavelength Translation in All-Optical Networks," in Proc. IEEE INFOCOM, March 1996, pp. 954-961

[30] A. Zalesky, H. L. Vu, Z. Rosberg, E. W. M. Wong, M. Zukerman, "Modelling and Performance Evaluation of Optical Burst Switched Networks with Deflection Routing and Wavelength Reservation," in Proc. IEEE INFOCOM, Hong Kong, China, March 2004.

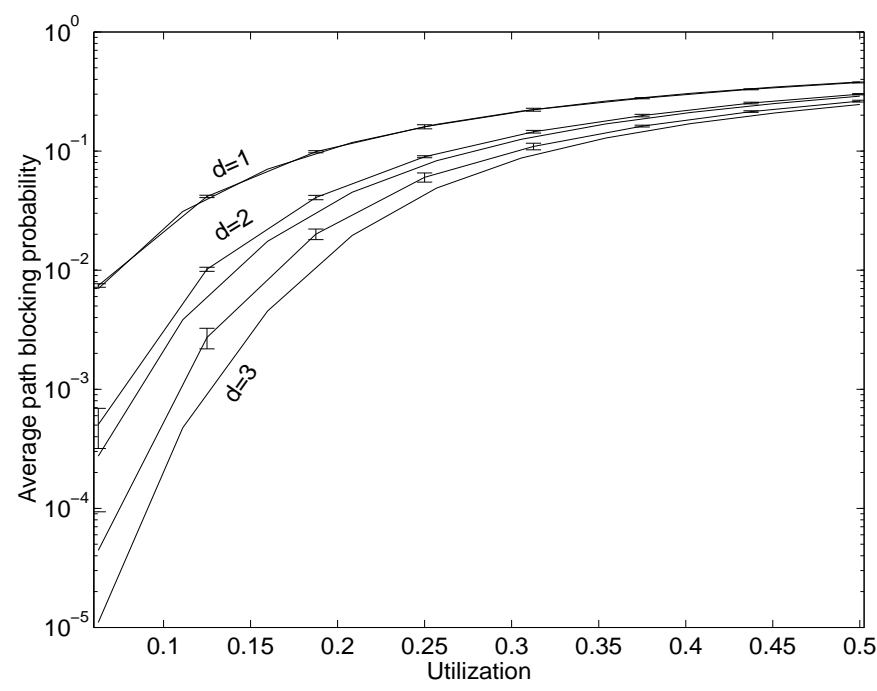

Fig. 6. Average path blocking probability estimated by framework and simulation for set 1 with neither segmentation nor deflection

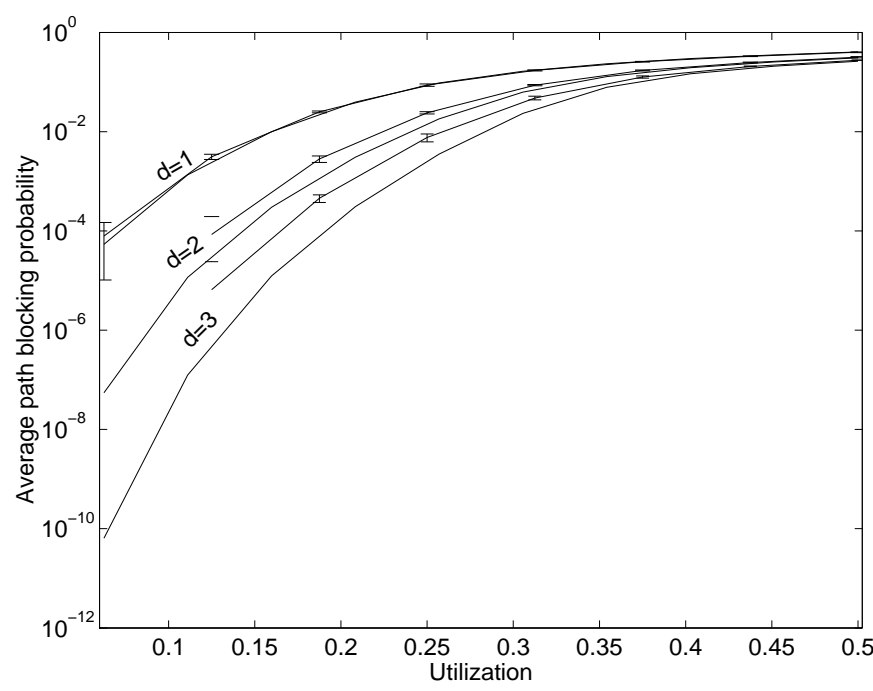

Fig. 7. Average path blocking probability estimated by framework and simulation for set 1 with deflection 


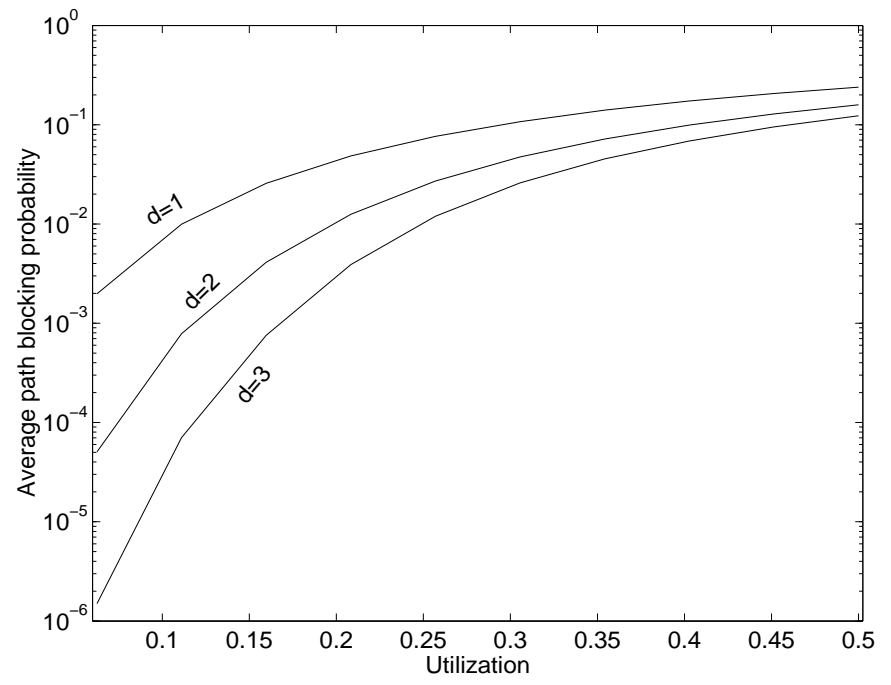

Fig. 8. Average path blocking probability estimated by framework for set 1 with segmentation

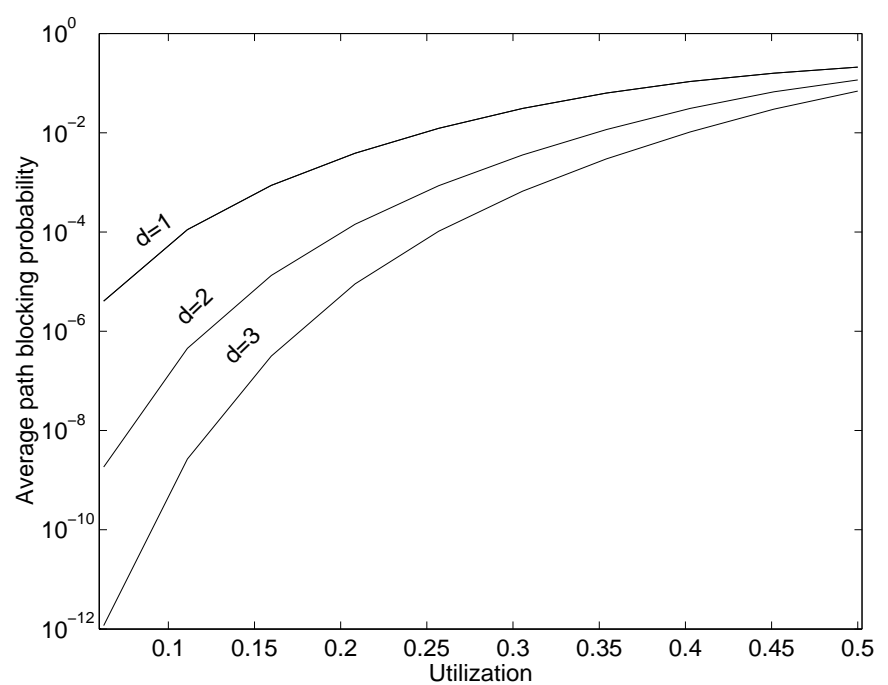

Fig. 9. Average path blocking probability estimated by framework for set 1 with segmentation and deflection

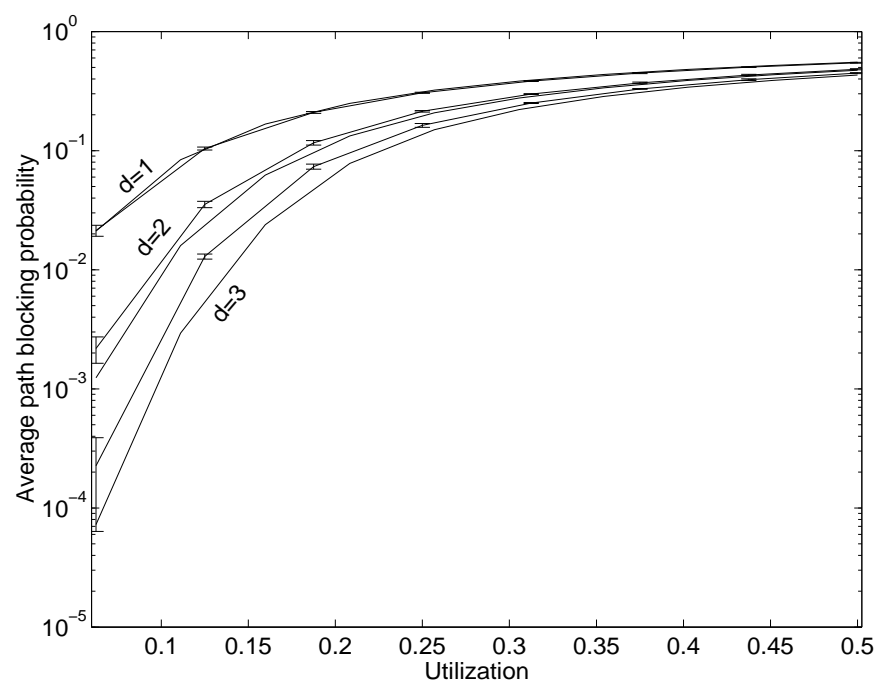

Fig. 10. Average path blocking probability estimated by framework and simulation for set 2 with neither segmentation nor deflection

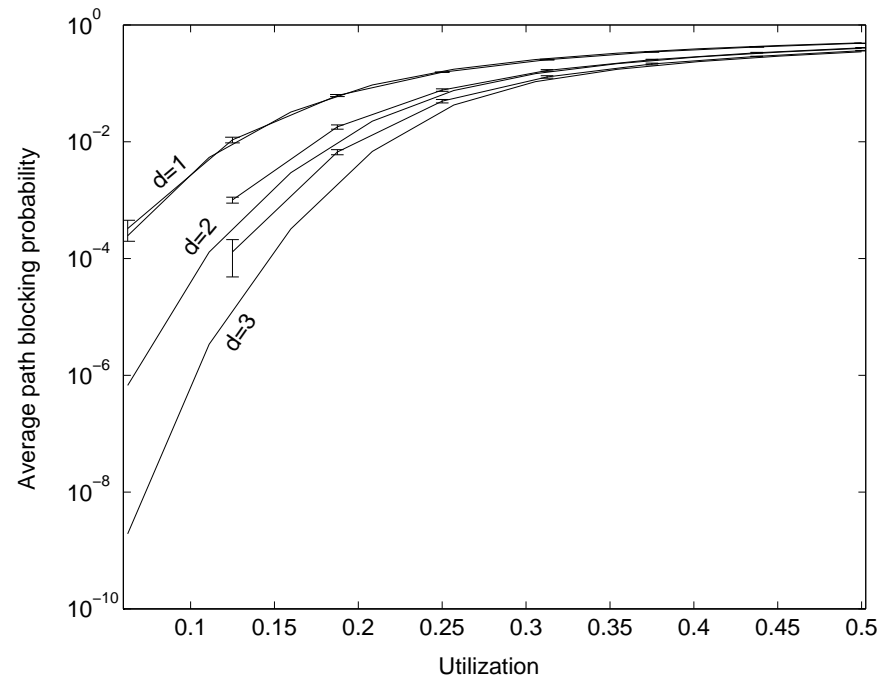

Fig. 11. Average path blocking probability estimated by framework and simulation for set 2 with deflection

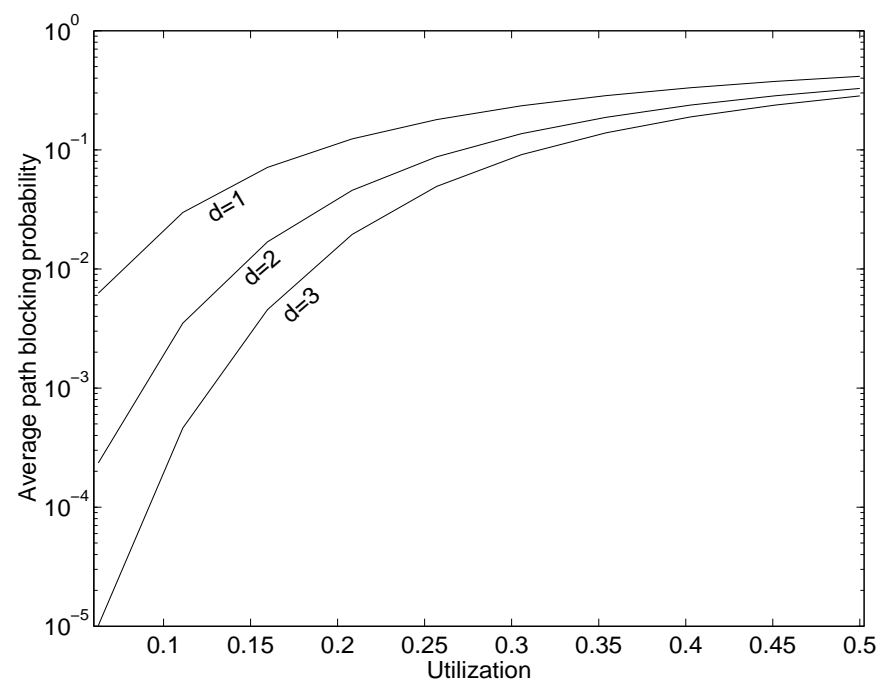

Fig. 12. Average path blocking probability estimated by framework for set 2 with segmentation

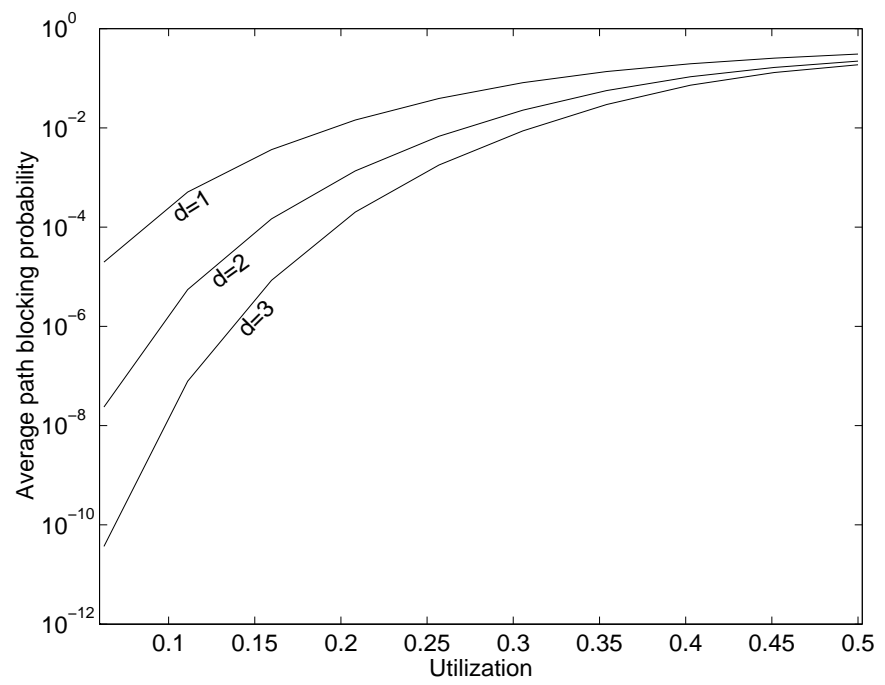

Fig. 13. Average path blocking probability estimated by framework for set 2 with segmentation and deflection 